\title{
Randomized Controlled Clinical Trial on Proximal Caries Infiltration: Three-Year Follow-Up
}

\author{
H. Meyer-Lueckela ${ }^{\mathrm{a}} \quad$ K. Bitter ${ }^{\mathrm{b}} \quad$ S. Paris ${ }^{\mathrm{c}}$
}

a Department of Operative Dentistry, Periodontology and Preventive Dentistry, Rheinisch-Westfälische Technische Hochschule Aachen (Aachen University), Aachen, ${ }^{b}$ Department of Operative Dentistry and Preventive Dentistry, Charité - Universitätsmedizin Berlin, Berlin, and ${ }^{\mathrm{C} C l i n i c ~ f o r ~ C o n s e r v a t i v e ~ D e n t i s t r y ~ a n d ~ P e r i o d o n t o l o g y, ~ S c h o o l ~ f o r ~}$ Dental Medicine, Christian-Albrechts-Universität zu Kiel, Kiel, Germany

\section{Key Words}

Caries infiltration • Caries lesion • Efficacy • Infiltrant •

Randomized controlled trial $\cdot$ Resin

\begin{abstract}
We report the 3-year efficacy of resin infiltration (Icon, preproduct; DMG, Hamburg) to arrest progression of proximal non-cavitated caries lesions as compared with placebo treatment. In 22 young adults, 29 lesion pairs with radiographic extensions into the inner half of enamel up to the outer third of dentin were included (split-mouth design). All subjects received risk-related instructions for diet, flossing and fluoridation. No unwanted effects could be observed. Radiographically $1 / 26$ test lesions (4\%) and $11 / 26$ control lesions (42\%) had progressed ( $p=0.002$, McNemar). After 3-year followup, infiltration of proximal caries lesions can be said to be efficacious to reduce lesion progression.
\end{abstract}

Copyright $\odot 2012$ S. Karger AG, Basel

For occlusal surfaces, caries sealing may fill the 'gap' between non-invasive and invasive (restorative) treatment options [Griffin et al., 2008]. This sealing concept has been adopted to proximal caries lesions [Gomez et al.,
2005; Martignon et al., 2006]. The aim of the caries infiltration technique is to allow penetration of a low-viscosity resin into the porous lesion body of enamel caries [Meyer-Lueckel and Paris, 2008; Paris et al., 2010; Paris and Meyer-Lueckel, 2010]. Hereby diffusion pathways for cariogenic acids are blocked and lesions are sealed inside and not on their surfaces, which facilitates clinical application especially in the proximal space [Phark et al., 2009], since no temporary tooth separation [Pitts and Longbottom, 1987] is required.

The efficacy of caries infiltration after up to 3 years follow-up has so far been reported in three studies using a split-mouth design. In young children with moderate to high caries risk, $23 \%$ of infiltrated lesions in primary molars showed progression, whereas $62 \%$ of the control lesions progressed radiographically within 1 year. On both test and control lesions fluoride varnish had been applied at baseline and after 6 months [Ekstrand et al., 2010]. Recently, 3-year follow-up progression rates of 32\% (test) and 70\% (control) were reported [Martignon et al., 2012]. The first evaluation after 18 month of the present study population revealed progression of $7 \%$ and $37 \%$ of the test and control lesions, respectively [Paris et al., 2010].

The aim of the present study was to assess the efficacy of resin infiltration of proximal caries lesions for this

\section{KARGER}

Fax +4161306 1234

E-Mail karger@karger.ch

www.karger.com
(C) 2012 S. Karger AG, Basel

0008-6568/12/0466-0544\$38.00/0

Accessible online at:

www.karger.com/cre
PD Dr. Sebastian Paris

Clinic for Conservative Dentistry and Periodontology

School of Dental Medicine, Christian-Albrechts-Universität zu Kiel

Arnold-Heller-Strasse 3, Haus 26, DE-24105 Kiel (Germany)

Tel. +49 431597 2817, E-Mail paris@konspar.uni-kiel.de 
population after 3 years. It was hypothesized that the radiographic lesion progression of infiltrated proximal lesions would be significantly reduced compared with noninfiltrated control lesions.

\section{Materials and Methods}

The study design was a split-mouth placebo-controlled randomized clinical trial. Ethical approval was given by the local institutional board at Charité - Universitätsmedizin Berlin (EA4/053/07). The methodology has been thoroughly described in conjunction with the 18-month efficacy data presented previously [Paris et al., 2010]. Therefore, we only repeat the most important methodological aspects within the current publication.

After screening (visual-tactile caries assessment) for general eligibility, a pair of standardized conventional bitewing radiographs was taken using individualized holders in order to allow reproducible alignment of the holder. From 61 screened subjects, 22 patients met the inclusion criteria and gave informed consent. For each patient one (15 patients) or two pairs (7 patients) of non-cavitated proximal caries lesions (as assessed with a thin probe without prior tooth separation) with the radiographic extensions from the inner half of enamel (E2) up to the outer third of dentin (D1) were selected [Hintze et al., 1999]. Either one caries lesion of each pair was allocated randomly to the infiltration or to the placebo group, respectively. Papilla bleeding was assessed and lesion surface characteristics were recorded. Caries risk of included patients was assessed using the Cariogram software [Bratthall and Hansel Petersson, 2005]. In contrast to baseline evaluation for the 3 -year caries risk evaluation, no salivary tests were performed (i.e. mutans streptococci count, buffer capacity and stimulated flow rate).

Infiltration (test) and placebo treatment (control) was performed by H.M.L. as described previously [Paris et al., 2010], patients being blinded to lesion allocation throughout the whole study period as a placebo treatment was performed on the control lesions, i.e. acid and infiltrant were not applied, but the control area was manipulated in a similar way and length as for the infiltration procedure of the test lesion. Patients were instructed to floss all proximal areas at least twice a week and to regularly use fluoride toothpaste. According to individual caries risk, additional fluorides sources were recommended. General oral hygiene education and dietary advice was given as well. A card was handed to patients to inform external dentists about the participation in the study and about identification of selected teeth.

After 18 months [Paris et al., 2010] and 36 months (this paper), follow-up examinations were performed by one clinical investigator (K.B.), who was blinded with regard to treatment allocation of teeth. Patients were interviewed for possible unwanted effects. The primary endpoint was lesion progression as assessed by digital subtraction radiography (DSR). Secondary endpoint was lesion progression by pairwise comparison (PW) of the X-rays. If lesions had progressed radiographically up to D2 or D3 stage, they were referred to restorative treatment. Standardized bitewing radiographs were obtained using individualized holders and assessed by two examiners (S.P. and K.B., both blinded for treatment allocation of teeth) as described previously. In case of differing interpretation between the examiners a consensus rank was agreed on.

Randomized Controlled Clinical Trial on Proximal Caries Infiltration
Sample size calculation as well as 'almost perfect' inter- and intrarater reliability (kappa values) had been reported for DSR in the 18-month evaluation [Paris et al., 2010]. Differences in number of progressing lesions as between test and control as assessed by PW and DSR were analyzed using the McNemar test. As a sensitivity analysis in order to address the choice of two lesion pairs within one subject we randomly excluded seven lesion pairs in the respective patients.

\section{Results}

The mean (SD) chance of avoiding new caries lesions [chance to avoid new caries $=1-$ caries risk $=54 \%(15 \%)$ ] was similar to baseline [60\% (22\%)]. Patients showed lower mean plaque index $[28 \%(11 \%)]$ compared with baseline $[38 \%(21 \%)]$ and reported to have followed recommendations regarding fluoridation. Patients did not report any complaint or unwanted effects. Also clinically no unwanted effects could be observed.

After 3 years (September 2010), 19 out of 22 participants having 25/29 lesion pairs were followed up clinically and radiographically. Additionally one patient having two lesion pairs could be followed up after 4.3 years. One patient with one lesion pair was not available for the present followup. One patient with one lesion pair had dropped out of the analyses at the 18-month follow-up. For one patient with initially two lesion pairs, one lesion pair dropped out of the analysis because both the control and the test lesion had been restored by another dentist during the last 18 months, since the control lesion had shown signs of irreversible pulpitis (pain on cold stimuli) as reported by the patient. Both lesions did not show progression at the 18-month followup; the second lesion pair did not progress after 3 years. After 18 months, in one patient with one lesion pair both test and control lesions had progressed and been referred to restorative treatment. Thus, in total 26/29 lesion pairs in 20/22 patients were included for radiographic evaluation after 3 years. One control lesion was referred to restorative treatment, since it had progressed radiographically into the middle third of dentin. Results of reading lesion stage from bitewing radiographs are given in figure 1 .

PW of radiographs revealed that $1 / 26$ test lesions (4\%) and $9 / 26$ control lesions (35\%) had progressed $(\mathrm{p}=0.008$, McNemar). With DSR 1/26 lesions (4\%) had progressed in the test group compared with 11/26 lesions (42\%) in the control group ( $p=0.002$, McNemar) (fig. 2). In addition to the described changes of (radiographic) stages, we observed 2 and 4 lesions progressing (DSR evaluation) within the radiographic scores $\mathrm{E} 2$ and $\mathrm{D} 1$, respectively. The relative risk (95\% CI) for progression (DSR) for the con- 
Fig. 1. Change of radiographic lesion stages from baseline (here only E2 and D1 eligible) to follow-up at 3 years. Radiographic scores modified from Hintze et al. [1999].

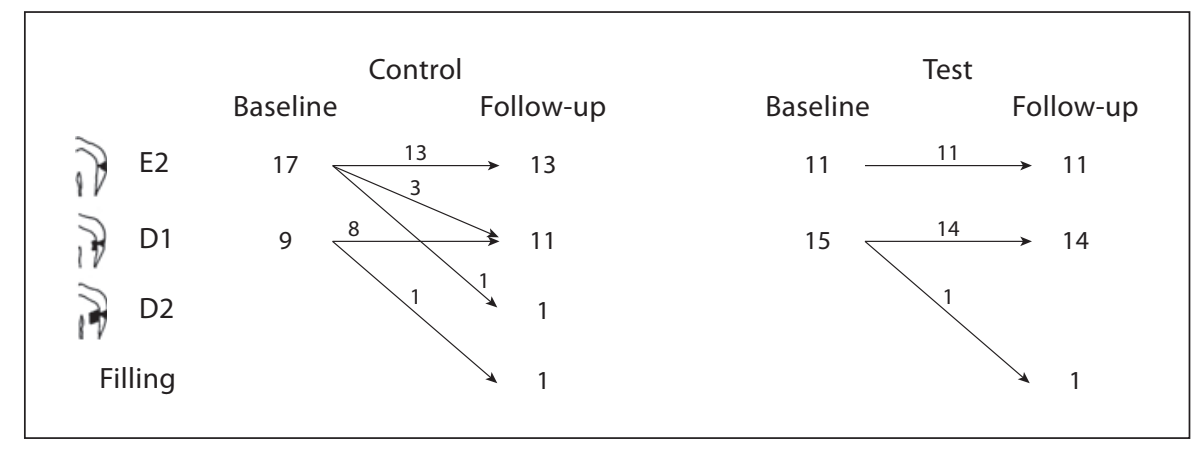

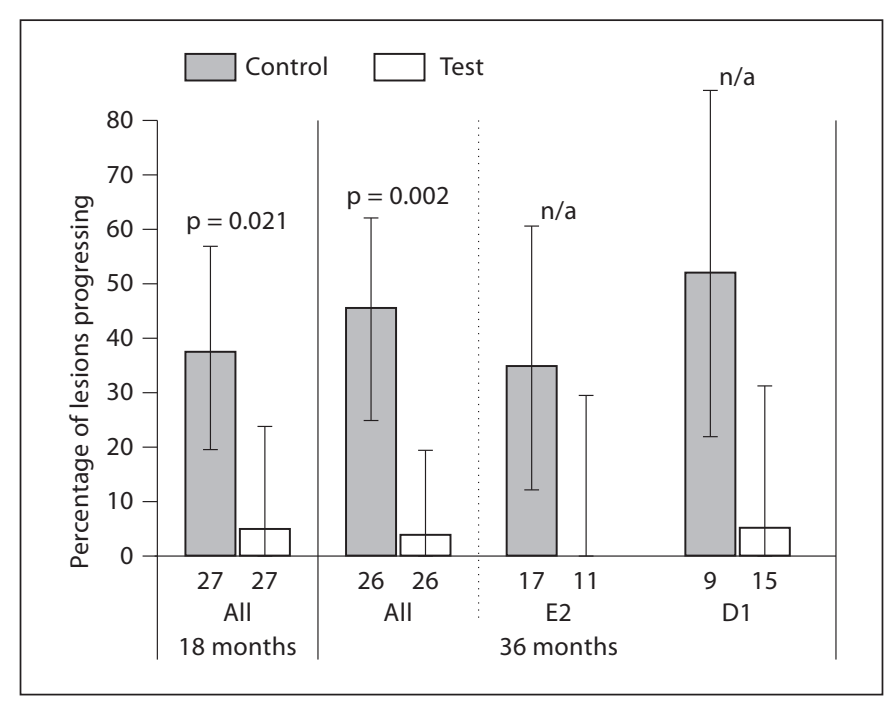

Fig. 2. Percentages of caries lesions progressing after 18 months [Paris et al., 2010] and 36 months that were either infiltrated (test) or not (control) evaluated by DSR (error bars $=95 \%$ Clopper-Pearson intervals; p values as calculated by the McNemar test). The stratified data according to baseline radiographic extension reveal a higher percentage of progressing control lesions for D1 compared with E2. $\mathrm{n} / \mathrm{a}=$ Not assessed.

trol compared with the test group was $11.0(1.8-231)$. When including the lesion pair that showed progression of the test lesion and restoration of the control lesion (decision by another dentist), the relative risk was calculated at 6.0 (1.5-38). No change from a higher radiographic stage at 18 months to a lower score at 36 months could be observed. Sensitivity analysis (exclusion of double lesion pairs in 7 patients) yielded $p$ values of 0.031 and 0.008 for PW and DSR evaluation, respectively. Here, the number of progressing infiltrated lesions was $1 / 20$ (both methods) as well as 7/20 (PW) and 9/20 (DSR) of the control lesions in the 20 individuals evaluated.

\section{Discussion}

It needs to be stressed that we did not use the clinical product (Icon; DMG, Hamburg) since it was not available when the study was initiated. Nonetheless, as discussed previously [Paris et al., 2010], compared to the commercial product only the applicator was different, which is considered to be of subordinate importance. Moreover, a longer application time of the infiltrant of $5 \mathrm{~min}$ was chosen compared with the recommendations given in the product instructions. This should not have influenced the efficacy of the infiltration technique considerably, since similar penetration depths [Meyer-Lueckel et al., 2011] and inhibition of caries progression (unpublished results) could be observed in vitro when using a shorter application time of $3 \mathrm{~min}$. The evaluation of primary and secondary outcomes was performed analogous to our previous study to which we refer for the discussion of methodological radiographic issues. Concerning the statistical analyses one might argue that although carious progression between various locations in a mouth is strongly correlated and thus statistically dependent, the occurrence of a discordant pair is far less likely to be correlated within patients. Thus, although the analysis of all 26 pairs in 20 patients violates statistical independence of all analyzed pairs, we assume this as a minor statistical problem, which is corroborated by the sensitivity analysis (random choice of 20 pairs in 20 patients) showing similar risk estimates as the primary analysis including all 26 pairs evaluated.

The population studied (mean baseline DMFT at age $25=6.7$ ) was supposed to be representative for other countries with developed market economies [Holst and Schuller, 2000; Mejare et al., 2004]. As indicated by the averaged medium caries risk some caries incidence was expected, which could be confirmed after 3 years (mean DMFT $=9.4$ ). It should be noticed that only three lesions 
showed radiological progression into the middle third of dentin indicating a need for restoration. This supports the philosophy of late invasive intervention, although the progressions observed from E2 to D1 and within D1 should also be noticed. Obviously, even in this rather low to medium caries risk group a high proportion of caries lesions progressed at least slightly, although it can be speculated that adherence to oral hygiene regimes should have been above the average of the German population as assessed by the caries risk parameters. Most interestingly, caries incidence after 18 and 36 months was very similar, which supports that caries is mainly observable in a small proportion of a young adult population [Mejare et al., 2004].

In the aforementioned study in young children with higher caries risk (12 months follow-up), the efficacy of resin infiltration in deciduous molars was not as high as in the present study [Ekstrand et al., 2010]. Besides the reported higher caries risk several other facts might have contributed to this result. First, a larger proportion of D1 lesions had been included in the study on children (60\%) compared with our investigation (44\%). This also holds true for the study on infiltration with 3-year follow-up (62\%), which may partially explain the overall higher progression rates and the comparatively lower efficacy of the infiltration technique [Martignon et al., 2012]. Although in all three studies operators aimed at choosing non-cavitated caries lesions only, the probability of including (micro-)cavitated lesions increases with higher proportions of deeper lesions [Pitts and Rimmer, 1992; Hintze et al., 1998]. These will show incomplete infiltration of the cavitated areas [Paris et al., 2011] and in turn be more likely to progress compared with non-cavitated ones having similar radiographic extension. Moreover, in the study on deciduous teeth, no individualized bitewing plates were used [Ekstrand et al., 2010], which bears the risk of higher numbers of caries lesions being false-positively judged as progressive. This would have resulted in higher proportions of progression in both test and control lesions, but would also have shifted the relative risk reduction in favor for the control lesions. Additionally, caries progression rate in deciduous molars is supposed to be higher compared to the rates in premolars and molars in young adults [Shwartz et al., 1984; Hintze et al., 1999; Mejare et al., 2004], which might have lowered the efficacy of caries infiltration as well.

The current results corroborate that caries infiltration is an efficacious method to hamper progression of noncavitated proximal lesions extending radiographically into the inner half of enamel up to the outer third of dentin after a period of 3 years. Therefore, this treatment seems to fill the 'treatment gap' between non-invasive and invasive interventions for proximal caries lesions.

\section{Acknowledgments}

The study was conducted at Charité - Universitätsmedizin Berlin, which is hereby acknowledged. The authors thank Dr. Christiane Nobel (Oral Surgery, Charité - Universitätsmedizin Berlin) for supporting the screening and Prof. Dr. Ralf-Dieter Hilgers (Institute of Medical Statistics, Aachen University) for statistical advice.

The study was supported by DMG, Hamburg. The funder had no role in study design, data collection and analysis, decision to publish or preparation of the manuscript.

H. Meyer-Lueckel and S. Paris conceived and designed the study; H. Meyer-Lueckel performed the clinical treatment; K. Bitter and S. Paris performed the clinical and radiographic assessment: H. Meyer-Lueckel and R.-D. Hilgers analyzed the data; H. Meyer-Lueckel and S. Paris wrote the paper.

\section{Disclosure Statement}

The study was supported by DMG, Hamburg, the producer of a commercial kit for caries infiltration. H. Meyer-Lueckel and S. Paris are appointed as inventors of US and European patents for an infiltration technique for dental caries lesions, held by Charité - Universitätsmedizin Berlin, and receive royalties from DMG.

\section{References}

Bratthall D, Hansel Petersson G: Cariogram - a multifactorial risk assessment model for a multifactorial disease. Community Dent Oral Epidemiol 2005;33:256-264.

-Ekstrand KR, Bakhshandeh A, Martignon S: Treatment of proximal superficial caries lesions on primary molar teeth with resin infiltration and fluoride varnish versus fluoride varnish only: efficacy after 1 year. Caries Res 2010;44:41-46.
Gomez SS, Basili CP, Emilson CG: A 2-year clinical evaluation of sealed noncavitated approximal posterior carious lesions in adolescents. Clin Oral Investig 2005;9:239-243.

- Griffin SO, Oong E, Kohn W, Vidakovic B, Gooch BF, Bader J, Clarkson J, Fontana MR, Meyer DM, Rozier RG, Weintraub JA, Zero DT: The effectiveness of sealants in managing caries lesions. J Dent Res 2008;87:169174 .

Randomized Controlled Clinical Trial on

Hintze H, Wenzel A, Danielsen B: Behaviour of approximal carious lesions assessed by clinical examination after tooth separation and radiography: a 2.5-year longitudinal study in young adults. Caries Res 1999;33:415422. 
-Hintze H, Wenzel A, Danielsen B, Nyvad B: Reliability of visual examination, fibre-optic transillumination, and bite-wing radiography, and reproducibility of direct visual examination following tooth separation for the identification of cavitated carious lesions in contacting approximal surfaces. Caries Res 1998;32:204-209.

-Holst D, Schuller AA: Oral health changes in an adult Norwegian population: a cohort analytical approach. Community Dent Oral Epidemiol 2000;28:102-111.

Martignon S, Ekstrand KR, Ellwood R: Efficacy of sealing proximal early active lesions: an 18-month clinical study evaluated by conventional and subtraction radiography. Caries Res 2006;40:382-388.

-Martignon S, Ekstrand KR, Gomez J, Lara JS, Cortes A: Infiltrating/sealing proximal caries lesions: a 3-year randomized clinical trial. J Dent Res 2012;91:288-292.
-Mejare I, Stenlund H, Zelezny-Holmlund C: Caries incidence and lesion progression from adolescence to young adulthood: a prospective 15-year cohort study in Sweden. Caries Res 2004;38:130-141.

Meyer-Lueckel H, Chatzidakis A, Naumann M, Dörfer CE, Paris S: Influence of application time on penetration of an infiltrant into natural enamel caries. J Dent 2011;39:465-469.

Meyer-Lueckel H, Paris S: Improved resin infiltration of natural caries lesions. J Dent Res 2008;87:1112-1116.

Paris S, Bitter K, Naumann M, Dörfer CE, Meyer-Lueckel H: Resin infiltration of proximal caries lesions differing in ICDAS codes. Eur J Oral Sci 2011;119:182-186.

Paris S, Hopfenmuller W, Meyer-Lueckel H: Resin infiltration of caries lesions: an efficacy randomized trial. J Dent Res 2010;89:823826.
Paris S, Meyer-Lueckel H: Infiltrants inhibit progression of natural caries lesions in vitro. J Dent Res 2010;89:1276-1280.

Phark JH, Duarte S Jr, Meyer-Lueckel H, Paris S: Caries infiltration with resins: a novel treatment option for interproximal caries. Compend Contin Educ Dent 2009;3:13-17.

Pitts NB, Longbottom C: Temporary tooth separation with special reference to the diagnosis and preventive management of equivocal approximal carious lesions. Quintessence Int 1987;18:563-573.

Pitts NB, Rimmer PA: An in vivo comparison of radiographic and directly assessed clinical caries status of posterior approximal surfaces in primary and permanent teeth. Caries Res 1992;26:146-152.

Shwartz M, Grondahl HG, Pliskin JS, Boffa J: A longitudinal analysis from bite-wing radiographs of the rate of progression of approximal carious lesions through human dental enamel. Arch Oral Biol 1984;29:529-536. 